\title{
Mathematical theory of peer-instruction dynamics
}

\author{
Hideo Nitta \\ Department of Physics, Tokyo Gakugei University, Koganei, Tokyo 184-8501, Japan
}

(Received 31 March 2010; published 3 August 2010)

\begin{abstract}
A mathematical theory of peer instruction describing the increase of the normalized number of correct answers due to peer discussion is presented. A simple analytic expression is derived which agrees with class data. It is shown that our theory is connected to the mathematical learning models proposed by Pritchard et al. It is also shown that obtained theoretical lines are useful for analyzing peer-instruction efficiencies.
\end{abstract}

DOI: 10.1103/PhysRevSTPER.6.020105

PACS number(s): 01.40.Fk, 01.40.Ha

\section{INTRODUCTION}

Recently, there has been a growing interest in constructing mathematical teaching-learning models [1-4]. Although the ultimate purpose of physics education research is to develop most effective instruction methods, a mathematical theory of learning is worth studying as far as the theory provides useful information for analyzing students' difficulties as well as improving instructions. Of course, it is unlikely that constructing quantitative theories of learning for individuals become possible, at least in the near future, because such theories would in fact describe student's history itself. However, when we consider the learning process for many students it may become possible to construct a theory that provides certain quantitative predictions. This is just like the fact that statistical mechanics based on a few basic laws and variables describes the properties of macroscopic systems composed of huge number of atoms and molecules, while in such a system the motion of individual atoms and molecules is so complex that it is extremely difficult to predict it in a precise manner.

In this paper we develop a phenomenological theory of peer instruction (PI) [5,6]. In contrast to the previous theories that describe long-term learning gain $[1,4]$, our theory describes rather a short-time process of learning. Nevertheless, as we will show in Sec. III B, our simplified analytic expression turns out to be connected to the mathematical model proposed by Pritchard et al. based on the constructivist view [4].

In Sec. II we start with a "master equation" describing the transition of the number of students answering correctly for a multiple-choice question during PI. We show that the number of correct answers after peer discussion is approximately given by a simple function of the number of correct answers before discussion. In Sec. III we compare our theory with class data. Also, comparison is made of our theory with learning models proposed by Pritchard et al. [4]. Applications of our theory to data analysis are discussed in Sec. IV. A few concluding remarks are given in Sec. V.

\section{THEORY}

Although there are variations in implementing PI $[7,8]$, in this report we only consider PI based on Mazur's original description [5]: students are expected to answer a concept oriented multiple-choice question (MCQ) individually before discussion.
We neglect many factors belonging to individual student such as personality, education, rhetorical abilities, personal skills, etc. We call them "student parameters." Under this condition, we may introduce distribution functions which represent the normalized number of students choosing the correct answer before and after discussion for a MCQ. Naturally, besides student parameters, these functions would depend on many variables such as the subjects of the lecture before posing MCQ, contents and quality of the MCQ, strength of psychological coupling with neighbors, etc. However, we consider only the indices of answers for the MCQ as explicit variables. Then, we may construct a "master equation" of PI for a MCQ that describes the number of correct answers before and after discussion. By denoting the normalized number of students choosing the answer $a$ for the MCQ $q$ before discussion and after discussion as $\rho_{1}(q ; a)$ and $\rho_{2}(q ; a)$, respectively, $\rho_{2}(q ; a)$ may be given by

$$
\rho_{2}(q ; c)=\rho_{1}(q ; c)-\sum_{d(\neq c)} T_{d c}(q) \rho_{1}(q ; c)+\sum_{d(\neq c)} T_{c d}(q) \rho_{1}(q ; d)
$$

where $c$ and $d$ represent the correct answer and distractors, respectively. The second term of the r.h.s. of Eq. (1) represents the "outgoing processes" in which students who polled the correct answer before discussion change their responses to distractors after discussion. The third term represents the "incoming processes" in which students who polled distractors before discussion change their responses to the correct answer after discussion. $T_{a b}(q)$ is the "transition matrix" which represents the normalized transition rate of students from answer $b$ to $a$. In general, such a transition rate is a function of $\rho_{1}(q ; c)$, student parameters, student-student relationship, contents of posed MCQs, and many other variables. $T_{a b}(q)$ satisfies the condition $0 \leq T_{a b}(q) \leq 1$ and, in general, $T_{a b}(q) \neq T_{b a}(q)$. It should be noted that $\rho_{1}(q ; c)$ and $\rho_{2}(q ; c)$ do not coincide with the precise numbers of students having the relevant knowledge. Actually, students choose the correct answer in variety of confidence levels [5] and sometimes based on incorrect reasoning.

Although Eq. (1) is already a simplified expression in that we have neglected many factors affecting $\rho_{1}(q ; a)$ and $\rho_{2}(q ; a)$, let us try to simplify it further for the purpose to find an analytic formula. We assume that the transition rates from the correct answer to distractors are small, i.e., $T_{d c}(q)$ 
$\ll 1$ for all $d$, and that the transition rates from distractors to the correct answer do not differ very much, i.e., $T_{c d}(q)$ $\approx \bar{T}(q)$, for all $d$. Then Eq. (1) reduces to

$$
\rho_{2}(q ; c)=\rho_{1}(q ; c)+\bar{T}(q)\left[1-\rho_{1}(q ; c)\right],
$$

where we have used the identity $\Sigma_{d(\neq c)} \rho_{1}(q ; d)=1-\rho_{1}(q ; c)$. We assume further that $\bar{T}(q)$ can be expanded into the power series in $\rho_{1}(q ; c)$ as

$$
\begin{aligned}
\bar{T}(q)= & k_{0}(q)+k_{1}(q) \rho_{1}(q ; c)+k_{2}(q) \rho_{1}(q ; c)^{2}+\cdots \\
& +k_{n}(q) \rho_{1}(q ; c)^{n}+\cdots .
\end{aligned}
$$

Let us take the linear approximation for Eq. (3). Since the transition to the correct answer is negligible when $\rho_{1}(q ; c)$ $\rightarrow 0$, we may assume that $k_{0}(q) \approx 0$. Then we obtain

$$
\bar{T}(q) \approx k_{1}(q) \rho_{1}(q ; c) .
$$

Equation (4) denotes that the transition rate from distractors to the correct answer due to PI is approximately proportional to the number of student polling the correct answer before discussion. Substituting Eq. (4) into Eq. (2), we obtain

$$
\rho_{2}(q ; c)=\rho_{1}(q ; c)+k_{1}(q) \rho_{1}(q ; c)\left[1-\rho_{1}(q ; c)\right] .
$$

It is useful to introduce a quantity representing the "peerinstruction efficiency" (PIE) for a MCQ $q$,

$$
\eta(q) \equiv \frac{\rho_{2}(q ; c)-\rho_{1}(q ; c)}{1-\rho_{1}(q ; c)} .
$$

It should be noted that PIE resembles the Hake gain [9] in its definition but not in character. The Hake gain represents the learning gain for a series of lectures, while PIE represents the efficiency of students' discussion for a certain MCQ.

Using Eq. (2), we obtain,

$$
\eta(q)=\bar{T}(q) \text {. }
$$

Now, let us try to simplify Eq. (5) further by ignoring the dependence on the MCQ (i.e., the parameter $q$ ). For simplicity, from now on we do not write the variable $c$ explicitly. From Eq. (5), we observe that $\rho_{2}=1$ when $\rho_{1}=1$. Therefore, by considering the condition that for $\rho_{1} \rightarrow 1$ the PIE should approach to unity, we have $k_{1}=1$. Then Eq. (5) reduces to

$$
\rho_{2}=\rho_{1}+\rho_{1}\left(1-\rho_{1}\right),
$$

and the PIE becomes

$$
\eta=\rho_{1}
$$

\section{COMPARING THEORY WITH DATA AND OTHER MODELS}

\section{A. Comparing with class data}

In this subsection, we compare Eq. (8) and (9) with data obtained from lectures implementing PI [10]. In Fig. 1 we show the fraction of correct answers before and after peer discussion. This type of chart was originally given by Mazur $[5,11]$. The red solid curves represent the numerical value of
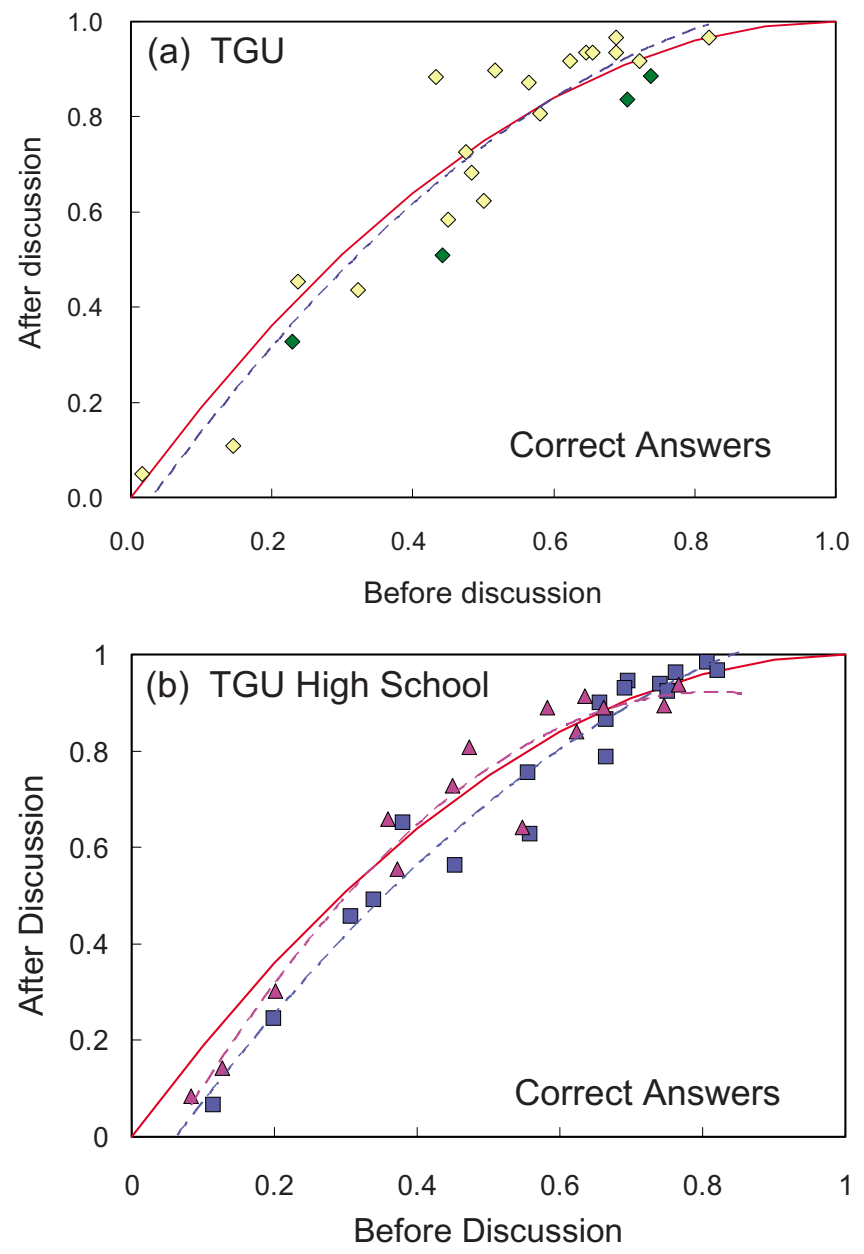

FIG. 1. (Color) The normalized number of correct answers after discussion versus before discussion for (a) a university course of introductory mechanics and (b) high-school physics courses. The red solid lines represent the theoretical curve of Eq. (8). In (a), the blue broken line represents the best fitting curve in the second-order polynomial approximation. The four green points represent data associated with Newton's third law. In (b) the violet and blue points correspond to the data from 2008 classes and 2009 classes, respectively. The violet and blue broken lines are corresponding best fitting curves.

Eq. (8). The data shown in Fig. 1(a) is obtained from the stand-alone type of lecture on introductory mechanics conducted by the author at Tokyo Gakugei University (TGU) in the spring semester of 2009 [12]. The blue broken line represents the second-order polynomial best fitting curve to data points by the least-squares method, which is given by $\rho_{2}$ $=-0.969 \rho_{1}^{2}+2.08 \rho_{1}-0.062$. Taking into account the fact that Eq. (8) is a result of drastic simplifications, the agreement between theory and data is well.

Figure 1(b) shows data obtained from high-school physics courses at Tokyo Gakugei University High School. The violet and blue data points are obtained by two instructors at 2008 and 2009 lectures on mechanics in the physics courses, respectively [13-15]. The violet and blue broken lines are best fitting curves to 2008 and 2009 data points, respectively. Our theory agrees with these high-school data. 

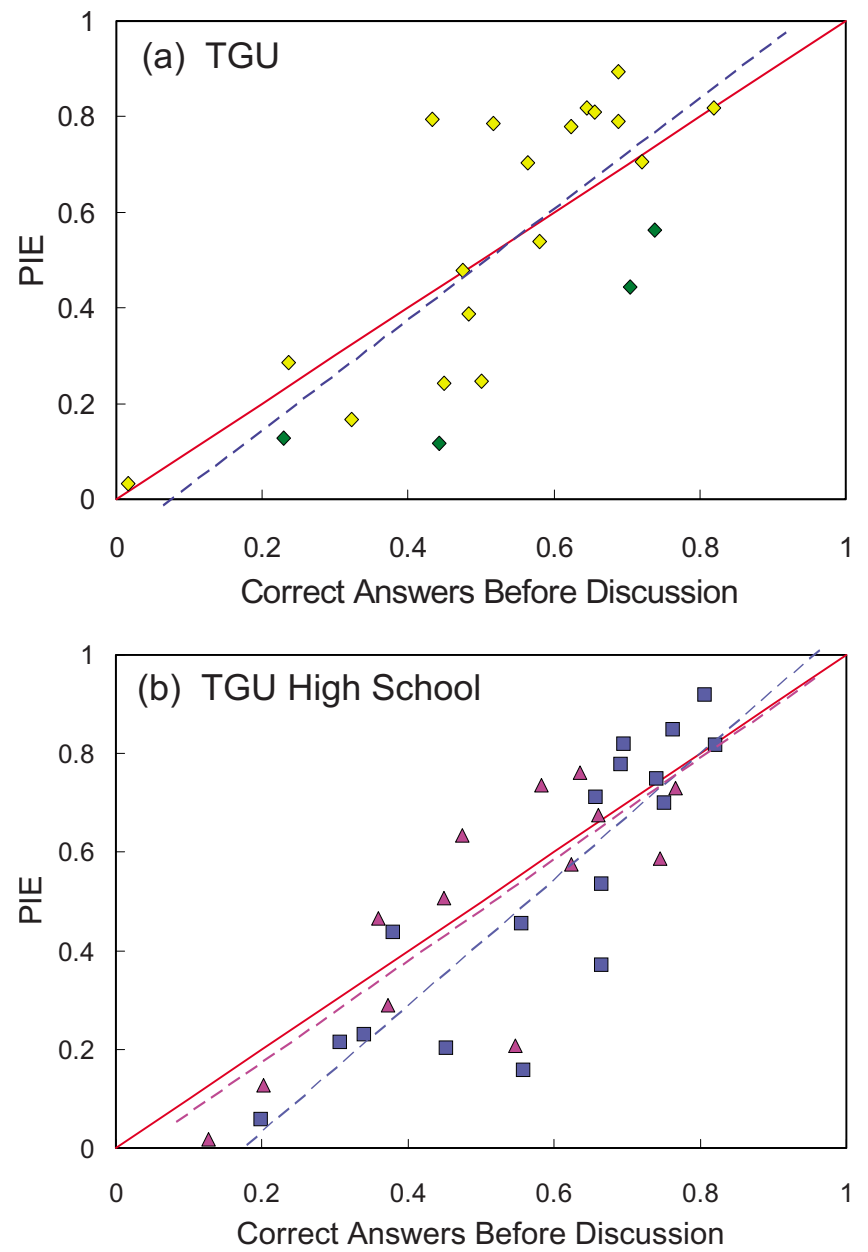

FIG. 2. (Color) The peer instruction efficiency (PIE) versus correct answers before discussion for (a) the university course and (b) the high-school courses. The red solid lines represent the theoretical line of Eq. (9). The broken lines represent the best fitting lines for the data. In (b) the violet (blue) points and line represent the 2008 (2009) data. The four green points in (a) represent data associated with Newton's third law.

In Fig. 2 we compare theoretical line of PIE [Eq. (9)] with data. The agreement between theory and data are again observed. As seen from Eq. (7), PIE is equivalent to $\bar{T}(q)$. Therefore, the linear dependence of PIE data on $\rho_{1}$ indicates the validity of the linear approximation for $\bar{T}$.

\section{B. Comparison with other models}

In this subsection we compare our theory with the mathematical models of learning developed by Pritchard et al. [4].

Let us take the r.h.s. of Eq. (3) up to the first order of $\rho_{1}$, i.e., $\bar{T}=k_{0}+k_{1} \rho_{1}$. Then substituting $\rho_{1}$ and $\rho_{2}$ by $\rho_{t}$ and $\rho_{t+\tau}$, respectively, we obtain from Eq. (2)

$$
\rho_{t+\tau}=\rho_{t}+\left(k_{0}+k_{1} \rho_{t}\right)\left(1-\rho_{t}\right) \text {. }
$$

Taking the limit $\tau \rightarrow 0$ for Eq. (10), we obtain the nonlinear differential equation

$$
\frac{d \rho_{t}}{d t}=\left(\alpha_{0}+\alpha_{1} \rho_{t}\right)\left(1-\rho_{t}\right)
$$

where we have defined that $\alpha_{0}=k_{0} / \tau$ and $\alpha_{1}=k_{1} / \tau$. Equation (11) is equivalent with the differential equation representing the "connectedness model" [4]. Indeed, if we put $\alpha_{0}=0$ and replace $\rho_{t}$ with $K_{T}(t)$ in Eq. (11), we obtain the "simple connected model,"

$$
\frac{d U_{T}(t)}{d t}=-\alpha_{1} U_{T}(t) K_{T}(t)
$$

where $K_{T}(t)$ and $U_{T}(t)$ are the known and unknown fractions of a certain test domain $T$, respectively, which satisfy $K_{T}(t)$ $+U_{T}(t)=1$ [4]. Since Eq. (8) is identical to Eq. (10) with $k_{0}$ $=0$ and $k_{1}=1$, Eq. (8) corresponds to Eq. (12). In this sense the dynamics of PI expressed by Eq. (8) agrees with the simple connected model of Pritchard et al. It is worthwhile to mention that Pritchard et al. noted as "this (simple connected) model would apply to classes that receive instruction solely by peer to peer interactions" [4].

On the other hand, if we put $\alpha_{1}=0$, we obtain,

$$
\frac{d U_{T}(t)}{d t}=-\alpha_{0} U_{T}(t)
$$

which is called the "pure memory model" [4]. Naturally this model is rather different from the learning process of PI. Indeed, the pure memory model corresponds to the case for $\bar{T}=k_{0}$. In this case, as seen from Eq. (7), PIE becomes constant. This result clearly disagrees with the data shown in Fig. 2.

\section{DISCUSSION}

In this section we illustrate possible applications of our theory to the diagnostics of students' difficulties and improvement of instruction.

The four green points in Figs. 1(a) and 2(a) are results of MCQs associated with Newton's third law. It is remarkable that, although values of the normalized number of correct answers before discussion for these points range from 0.23 to 0.74 , all of them are placed below the theoretical lines [16]. This suggests that individual students tend not to change their views about Newton's third law by peer discussion. In other words, students' naive concept [11] on Newton's third law is rather concrete. Of course this is well-known inductive conclusion in physics education research. We would like to point out that the simple analysis based on our theory recovers this established knowledge in physics education research.

It should be emphasized that Eq. (8) is derived by neglecting the dependence on MCQs. Obviously, if we use only low PIE-value MCQs for PI such as green data points in Figs. 1(a) and 2(a), data points will stay below the theoretical curve of Eq. (8). By contrast, if we use only very effective (high PIE-value) MCQs for PI, data points will beyond the theoretical curve. In this sense, it may be possible to use the theoretical curve of Eq. (8) as a certain standard to evaluate students' difficulties as well as effective MCQs from practical data. 


\section{CONCLUDING REMARKS}

In this paper, we have presented a theory describing dynamics of peer instruction. We have obtained a simple analytic expression that gives the relation between the rate of correct answers before and after discussion. The theoretical curve agrees with data obtained from lectures implementing PI. It is also shown that our simplified expression corresponds to the simple connected model of Pritchard et al. [4]. It is indicated that our theoretical results will be useful to diagnose student difficulties through PI data as well as finding effective concept oriented questions for PI.
Finally, it is worthwhile to point out that all parameters and functions in Eq. (1) can be determined from PI data obtained easily by using "clickers." It is interesting to use the transition matrix $T_{a b}(q)$ for the analysis of student difficulties, but such tasks are beyond the scope of our present report.

\section{ACKNOWLEDGMENTS}

The author is grateful to H. Takahashi and M. Kaneta for many fruitful discussions on peer instruction. This work was partly supported by JSPS KAKENHI Grant No. 22500801.
[1] C. M. Bordogna and E. V. Albano, Theoretical Description of Teaching-Learning Processes: A Multidisciplinary Approach, Phys. Rev. Lett. 87, 118701 (2001).

[2] C. M. Bordogna and E. V. Albano, Simulation of social processes: application to social learning, Physica A 329, 281 (2003).

[3] L. Bao and E. F. Redish, Model analysis: Representing and assessing the dynamics of student learning, Phys. Rev. ST Phys. Educ. Res. 2, 010103 (2006).

[4] D. H. Pritchard, Y.-J. Lee, and L. Bao, Mathematical learning models that depend on prior knowledge and instructional strategies, Phys. Rev. ST Phys. Educ. Res. 4, 010109 (2008).

[5] E. Mazur, Peer Instruction: A User's Manual (PearsonPrentice Hall, New Jersey, 1997).

[6] C. H. Crouch and E. Mazur, Peer Instruction: Ten years of experience and results, Am. J. Phys. 69, 970 (2001).

[7] D. E. Meltzer and K. Manivannan, Transforming the lecturehall environment: The fully interactive physics lecture, Am. J. Phys. 70, 639 (2002).

[8] C. Turpen and N. D. Finkelstein, Not all interactive engagement is the same: Variations in physics professors' implementation of Peer Instruction, Phys. Rev. ST Phys. Educ. Res. 5, 020101 (2009).

[9] R. R. Hake, Interactive-engagement versus traditional methods: A six-thousand-student survey of mechanics test data for introductory physics courses, Am. J. Phys. 66, 64 (1998).

[10] The TGU class was composed of 62 students. The appearance rate for the class was very good. In Fig. 1(a), the numbers of polling students are 55 for two points, 58 for one point, and more than 60 students for the rest of 20 points. The TGU high-school data are given as the sum of three classes for each year. A class is composed of about 45 students. All classes were given essentially the same lectures. The PI data in the Fig. 1(b) are the sum of 110 to 127 responses of students. The fluctuation of the responses is due to the different number of appearance to the class and of imperfect responses: responses only before or after PI have not been included in our data.

[11] E. F. Redish, Teaching Physics with the Physics Suite (Wiley, New York, 2003).

[12] In Japan an academic year starts from April. Usually, a lecture of $90 \mathrm{~min}$. is given weekly. A semester course is composed of 15 lectures.

[13] M. Kaneta and H. Nitta, Practice of peer instruction by use of clickers, Phys. Educ. Soc. Jpn. 57, 103 (2009), in Japanese.

[14] H. Takahashi and H. Nitta, A class of mechanics by adopting the peer instruction, Phys. Educ. Soc. Jpn. 57, 297 (2009), in Japanese.

[15] H. Takahashi and H. Nitta, Peer Instruction in Japanese High School Physics, in International Conference on Physics Education: ICPE-2009, edited by B. Paosawatyanyong and P. Wattanakasiwich, AIP Conf. Proc. No. 1263 (AIP, New York, 2010).

[16] In Fig. 2(a), 11 points lie above the theoretical line and 12 points below the line. Therefore, the probability that all of randomly selected four points from among those 23 points lie below the theoretical curve is 0.037 . 